\title{
Warum wir uns keine Ungerechtigkeiten mehr leisten können
}

In seinem jüngst erschienenen Buch „Die Idee der Gerechtigkeit“ betont Amartya Sen, „nicht die Erkenntnis, dass die Gerechtigkeit auf der Welt unvollkommen ist, treibt uns zum Handeln, sondern die Tatsache, dass es in unserer Umgebung Ungerechtigkeiten gibt. " Ein Grund dafür ist, dass das Ideal der Gerechtigkeit oftmals viel zu abstrakt ist und wir stattdessen viel klarere Vorstellungen darüber haben, was in einem konkreten Fall und in unserer unmittelbaren Umgebung ungerecht ist. Gerade die Vermeidung von Ungerechtigkeiten ist jedoch etwas, was in modernen Gesellschaften immer wichtiger wird. Denn zum einen beobachten wir eine zunehmende Sensibilität für sehr verschiedene Formen von Ungerechtigkeiten und zum anderen sind es die mit erfahrenen Ungerechtigkeiten verbundenen individuellen Reaktionen, die moderne wissensbasierte Gesellschaften schwächen können.

Um dies deutlich machen zu können, muss man sich zunächst vor Augen führen, warum Menschen überhaupt einen Sinn für Ungerechtigkeiten ausgebildet haben. Die Evolutionspsychologie verweist darauf, dass die bestehenden und sozial geteilten Vorstellungen über (Un-)Gerechtigkeit als eine Art Warnsystem funktionieren. Sie dienen dazu, im sozialen $\mathrm{Zu}$ sammenleben erkennen zu können, wann wir von anderen ausgenutzt oder ausgebeutet werden. Ausgangspunkt ist die spieltheoretische Einsicht, dass es in Kooperationszusammenhängen für jeden besser ist, wenn die anderen sich anstrengen und zur Herstellung eines gemeinsamen Gutes beitragen, man selbst aber keine Aufwendungen hat und trotzdem die Vorteile der Kooperation genießt. Freilich sind diejenigen, die ihren Beitrag leisten, die „Dummen“, da sie die Kosten tragen, während die anderen den Gewinn einstreichen. Und genau diese Ausbeutungssituation gilt es, zu vermeiden. Die in einer Gesellschaft oder Gruppe existierenden Regeln über eine gerechte Allokation und Distribution von Gütern und Lasten stellen einen Maßstab bereit, um solche Situationen zu erkennen. Denn sie bestimmen etwa, welche Belohnungen mit welchen Vorleistungen verknüpft sein sollten. Werden diese Vorstellungen verletzt, so ist dies ein Hinweis darauf, dass sich Einzelne auf Kosten anderer Vorteile verschaffen. In dem Fall unternehmen sie etwa weniger Anstrengungen, beziehen aber die gleichen oder sogar höhere Einkommen, oder sie zahlen geringere oder gar keine Steuern als sie gerechterweise zahlen sollten, genießen aber trotzdem die Fülle staatlicher Wohltaten. Je häufiger dies in einem spezifischen Kooperationszusammenhang - einem Unternehmen oder auch einer Gesellschaft - geschieht, umso höher ist auch die Wahrscheinlichkeit, dass man selbst zu den Benachteiligten gehört. Erkennt man derartige Ungerechtigkeiten, so kann man sich vor dem Ausgenutztwerden durch andere schützen, indem man selbst keine Beiträge mehr leistet. Man kündigt die eigene Koope- rationsbereitschaft auf und versucht in gleicher Weise, auf Kosten der anderen zu leben. Wichtig ist dabei auch, dass die Befolgung von Regeln und Normen in einem Sozialverband in gleicher Weise von der Kooperationsbereitschaft des Einzelnen abhängt. Menschen folgen den Verhaltenserwartungen von Regeln und sozialen Normen deshalb, weil sie mit anderen auch in Zukunft zusammenleben und zusammenarbeiten möchten. Wahrgenommene Ungerechtigkeiten entscheiden deshalb nicht nur darüber, ob Menschen ihren Anteil an der Herstellung gemeinsamer Güter leisten oder sich für eine gemeinsame Sache engagieren, sondern auch darüber, ob sie sich an soziale Normen und Regeln halten.

Genau diese Verhaltenskonsequenzen wahrgenommener Ungerechtigkeit können auch empirisch beobachtet werden: Arbeitnehmer, die sich ungerecht entlohnt fühlen, reduzieren ihre Arbeitsleistung, sie machen Dienst nach Vorschrift, halten sich nicht an basale Verhaltensregeln und versuchen, ohne Rücksicht auf andere ihren eigenen Vorteil zu realisieren. Umgekehrt gilt: Wo Güter und Lasten als „fair“ verteilt wahrgenommen werden, beobachtet man mehr Kooperation und Engagement. Genau deshalb sind Ungerechtigkeiten in der modernen Arbeitswelt höchst dysfunktional. Denn Betriebe sind auf ein Verhalten ihrer Beschäftigten angewiesen, das sich eben nicht auf die bloße physische Präsenz und die Verrichtung wiederholter und automatisierter Handgriffe erstreckt, sondern das durch freiwilliges Engagement, Kreativität und Kooperationsbereitschaft gekennzeichnet ist.

Zusammengenommen bedeutet dies: In dem Maße wie moderne Gesellschaften arbeitsteilig funktionieren, die alltäglichen Lebensvollzüge zunehmend in Kooperationszusammenhänge eingebunden und von den erzeugten Kooperationsergebnissen abhängig sind, steigt die Gefahr, von anderen ausgenutzt und ausgebeutet zu werden. Die in einer Gesellschaft bestehenden Vorstellungen über (Un-)Gerechtigkeiten dienen dem Einzelnen dazu, derartige Situationen zu erkennen und daraus Konsequenzen für die eigene Kooperationsbereitschaft zu ziehen. Die Abwesenheit von Ungerechtigkeit stellt die Voraussetzung für ein auf Kooperation und freiwilliges Engagement ausgerichtetes Verhalten dar. Nicht nur aufgrund der moralischen Forderung, die Welt besser zu machen, sind Ungerechtigkeiten deshalb zu vermeiden, sondern auch, weil wir aus ökonomischen Gründen darauf angewiesen sind, dass Menschen sich für gemeinsame Belange einsetzen und freiwilliges Engagement zeigen.

\footnotetext{
Stefan Liebig ist Professor für Soziologie mit dem Schwerpunkt Soziale Ungleichheit und Sozialstrukturanalyse an der Universität Bielefeld und Forschungsprofessor am Deutschen Institut für Wirtschaftsforschung (DIW). e-mail: stefan.liebig@uni-bielefeld.de
} 